Review Article

\title{
Involvement of the Circadian Rhythm and Inflammatory Cytokines in the Pathogenesis of Rheumatoid Arthritis
}

\author{
Kohsuke Yoshida, ${ }^{1}$ Teppei Hashimoto, ${ }^{2}$ Yoshitada Sakai, ${ }^{3}$ and Akira Hashiramoto ${ }^{1,4}$ \\ ${ }^{1}$ Faculty of Health Sciences, Kobe University School of Medicine, Kobe 654-0142, Japan \\ ${ }^{2}$ Department of General Internal Medicine, Kobe University School of Medicine, Kobe 650-0017, Japan \\ ${ }^{3}$ Division of Rehabilitation Medicine, Kobe University Graduate School of Medicine, Kobe 650-0017, Japan \\ ${ }^{4}$ Clinical Immunology, Kobe University Graduate School of Health Sciences, 7-10-2, Tomogaoka, Suma, Kobe 654-0142, Japan \\ Correspondence should be addressed to Akira Hashiramoto; hash@kobe-u.ac.jp
}

Received 5 March 2014; Accepted 24 April 2014; Published 8 May 2014

Academic Editor: Mizuko Mamura

Copyright (C) 2014 Kohsuke Yoshida et al. This is an open access article distributed under the Creative Commons Attribution License, which permits unrestricted use, distribution, and reproduction in any medium, provided the original work is properly cited.

Among the symptoms of patients with rheumatoid arthritis (RA), joint stiffness is influenced by diurnal rhythm and reaches peak in the morning, which is a common complaint and reflects the circadian nature of disease manifestation. In addition, inflammatory cytokines, which reach peak secretion early in the morning are major players causing the morning stiffness. In this review, we explore the link between the circadian clock and inflammation, focusing on the interactions of various clock genes with the immunepathways underlying the pathology of rheumatoid arthritis.

\section{The Biological Origins and Regulation of the Body Clock}

The word "circadian" is defined as the period of physiological function and behavior of the organism, which is about 24 hours in an environment with no external constraints. The circadian rhythm has been shown to be involved in a number of physiological processes, including sleep/awakening, body temperature regulation, hormone secretion, division and proliferation of cells, and gastrointestinal function. Although we normally think of the circadian rhythm as 24 hours, it is in fact slightly longer in humankind $[1,2]$. As a result, organisms need to correct their biological clock daily using external cues, the most effective of which is light stimulus. While other organisms use the same organ for both light reception and setting of the biological clock [3, 4], mammals have evolved separate locations for these two functions. The controlling center for rhythm oscillation in mammals is the hypothalamic suprachiasmatic nucleus (SCN), located at the base of the brain in front of the pituitary gland. The SCN is too deep in the brain to sense light cues directly and instead receives external cues via the optic nerve that transmits signals from the retina and combines the function of both light-dark tuning and rhythm transmission to act as a master clock of the whole body [5].

The role of the SCN in coordinating body rhythm was first shown in rodents, where removal of the SCN was observed to cause a collapse in the rhythmicity of behavior and endocrine activity, suggesting that this structure may be the underlying circadian pacemaker for physiological and behavioral activities in mammals [6]. However, subsequent studies found that the liver and lung cells maintain their own rhythm when cultured outside the body and without input from the SCN. Thus, it has become apparent that tissues and cells can provide their own peripheral rhythm, similar to that provided by the brain, which is maintained by the activities of the clock genes $[6,7]$.

The clock genes manage rhythm and time in a dual and hierarchical manner. The rhythm signals propagated from SCN are subject to a feedback loop provided by core clock genes including CLOCK (circadian locomotor output cycles kaput), BMAL1 (brain and muscle ARNTlike-1), PER (period) and CRY (cryptochrome), and orphan nuclear hormone receptors $R E V-E R B$ alpha (encoded by Nr1d1) and Ror alpha. The circadian expression of these genes is regulated through $\mathrm{E} / \mathrm{E}^{\prime}$ boxes (CACGTG/CACGTT 
sequence), REV-ERB $\alpha /$ ROR response element (RRE), and DBP/E4BP4 binding element (D box) in their promoter regions. First, the CLOCK and BMAL1 proteins form a complex that binds to the $\mathrm{E} / \mathrm{E}^{\prime}$ boxes present in the promoter regions of the Per1-3, Cry1-2, Nr1d1, and Ror alpha genes, thus regulating their transcription. The PER and CRY proteins, in turn, form a complex in the cytoplasm and relocate to the nucleus where they inhibit the genes induced by the CLOCK/BMAL1 protein complex, including their own transcription [6]. Second, REV-ERB and ROR compete on RRE element on the promoter region of $B M A L 1$ to regulate its transcription [8]. In addition, DBP (D site of albumin promoter binding protein) promotes and E4BP4 (E4-binding protein 4) suppresses the transcription of PER by binding to the $\mathrm{D}$ box present in the promoter region $[9,10]$. Although not all clock genes directly respond to CLOCK/BMAL1, this core control mechanism is repeated in about a 24hour period. Importantly, circadian transcriptional circuits are governed by two design principles [11]: regulation of $\mathrm{E} / \mathrm{E}^{\prime}$ boxes and RRE element follows a repressor-precedesactivator pattern, resulting in delayed transcriptional activity, whereas regulation of $\mathrm{D}$ box follows a repression-antiphasicto-activator mechanism, which generated high-amplitude transcriptional activity. In addition, recent study has shown that circadian modulation of RNA polymerase II recruitment and chromatin remodeling occurs on a genome-wide scale [12], so that there are various mechanisms for generating rhythmicity of gene expression.

\section{Melatonin and Inflammatory Cytokines Tune the Circadian Regulation}

In the daytime, light-initiated signals are transmitted via the SCN to the pineal gland through the upper cervical ganglion. Melatonin, a hormone that mediates circadian rhythm adjustment, is produced by the pineal gland at night. Light stimulus causes an increase in the secretion of cortisol, serotonin, and dopamine, while suppressing melatonin, norepinephrine, and acetylcholine. Serum melatonin levels are normally undetectable in the daytime but are significantly higher during the night, in the absence of optical stimulation [13-16].

How might melatonin be involved in the pathogenesis of rheumatoid arthritis? As compared with healthy subjects, melatonin secretion at midnight is significantly increased in RA patients [17], and melatonin serum levels in the morning are higher in RA patients with shorter disease duration [18]. Inflammatory cytokines including IFN- $\gamma$ (interferongamma), IL (interleukin)-1, and IL-6 are all secreted from human peripheral blood mononuclear cells in response to melatonin stimulation, and in fact, melatonin is detected in RA synovium tissue macrophages and joint fluid [19]. These studies seem to suggest that melatonin has an adverse effect on arthritis; on the other hand, melatonin inhibits the activity of MMP (matrix metalloproteinase)-9, which is involved in joint destruction in RA patients [20]. Thus, further study is needed to determine the effects of melatonin on joint destruction.
Studies in recent years have helped elucidate the influence of inflammatory cytokines on circadian mechanisms. For example, activation of the immune system counteracts infection and increases resistance to pathogens by inducing slow wave sleep, presumably via the production of inflammatory cytokines such as TNF- $\alpha$, IL-2, or IFN- $\gamma$ that are known to induce such sleep [21]. Indeed, LPS (lipopolysaccharide), which is the major component of the outer membrane of Gram-negative bacteria, stimulus also uniformly increases the secretion of these cytokines. Conversely, immune cells exhibit enhanced proinflammatory responses and LPSinduced IL- 6 release if the circadian rhythm is disrupted by an external change in the light-dark cycle [22]. Interestingly, LPS-dependent secretion of TNF- $\alpha$ is significantly higher at night compared to day and is further enhanced by melatonin stimulation. Through their ability to promote sleep, cytokines are involved in the generation of an inner rhythm that controls the secretion of growth hormone, prolactin, and cortisol [23].

How is the relation between sleep and rheumatoid arthritis that is well known to be a chronic inflammatory disease? RA patients often exhibit sleep disorders classified as a nocturnal awakening type, and this type of disorder is characterized by a significant reduction in sleep efficiency and a significant increase in waking periods after sleep onset. Questionnaire studies of patients with sleep disorders report a "decline in the quality of sleep in patients with RA" as quantified by the Pittsburgh Sleep Quality Index [24, 25], and "excessive somnolence trend during the day in patients with RA" by the Epworth sleepiness scale [26]. Further, increased RA disease activity has been reported to correlate with sleep disorders, and the correlation is somewhat stronger in women and is mitigated by age [27, 28]. In addition, shift work was associated with risk of RA in women [29]. This could be supported by the concept that the body clock not only impacts on arthritic symptoms but is also involved in the pathogenesis of RA [16].

In RA, a major source of proinflammatory cytokines is immune cells such as $\mathrm{T}$ cells and macrophages. Spleen, peripheral lymph nodes, and peritoneal fluid-derived macrophages operate autonomous circadian clockworks even ex vivo, and spleen cells secrete TNF- $\alpha$ and IL- 6 in a circadian manner under the stimulation with bacterial endotoxin [16, 30]. Further, endotoxin-induced cytokine and chemokine production was significantly affected by BMAL1 and REV$\mathrm{ERB} \alpha$ expression. Since the nuclear receptors $\mathrm{ROR} \alpha$ and $\mathrm{REV}-\mathrm{ERB} \alpha$ are key molecules that modulate BMAL1 transcription in the process of feedback-regulation of circadian genes, $R E V-E R B \alpha$ knockout condition represented the loss of circadian gating of endotoxin response, especially in the release of IL-6, through macrophages [31], and agonists or overexpression of REV-ERB $\alpha$ inhibited the expression of $\mathrm{Ccl} 2$, also named monocyte chemotactic protein-1 (MCP-1), by binding to their promoter regions in murine macrophage cell line [32]. In addition, REV-ERBs regulate their target genes by inhibiting the functions of distal enhancers that are 
selected by macrophage-lineage-determining factors, thereby establishing a macrophage-specific program of repression [33]. This relation between BMAL1 and inflammation was also demonstrated from different angles that BMAL1-deleted myeloid cells disrupted differentiation and circadian oscillation of inflammatory monocyte [34].

CD4 positive T cells possess a circadian oscillator that drives rhythmic responses to the stimuli, as manifested by altered cell proliferation and cytokine secretion [16]. Interestingly, it has been shown that $\mathrm{T}$ cell numbers and its reactivity were stable during daytime, whereas a significant increase was observed in the late evening and early morning hours ex vivo [35]. These results may explain the portion of etiology for "morning stiffness of joints", a common complaint and one of the best indicators of the condition of RA patients, correlates with the secretion of TNF- $\alpha$, IL- 6 , and IFN- $\gamma$, whose levels peak from midnight to early in the morning [36], and chronobiology-based approach has been tested and shown to improve the morning symptoms of RA [37, 38].

\section{A Pathological Link between RA and the Clock Genes: Synovitis and Wee-1 Kinase}

RA is a chronic polyarthritis condition that goes through repeated relapse and remission as the disease progresses. These cycles of elevated inflammation cause deformation and destruction of the joint and irreversible dysfunctions. All joints are affected by RA, large, medium and small. Once activated by inflammation, the mesenchymal cells lining the joint space (synovial cells) begin to proliferate, and inflammatory granulation tissue, called pannus, invades the bone and cartilage, leading to joint destruction. Growth factors, angiogenic factors, and inflammatory lymphocytes work together to promote pannus formation, and joint destruction follows the secretion of MMPs and migration of synovial cells [39, 40]. When we examine cytokine activities, we find that IL-1 $\beta$ and MMPs are deeply involved in osteoporosis of the joint and in the narrowing of the surrounding joint space seen in the early stages of disease onset. In the later stages, TNF- $\alpha$, IL$1 \beta$, and IL- 6 are produced and accumulate in the joint cavity as synovitis worsens. Finally, at the peak of arthritis, modification of the inflammatory response by IL-17 is observed [41].

However, lymphocytes are not present in the local pannus during the early stages of RA. In these early stages, joint destruction is promoted by synovial cells under the influence of various cell cycle regulators and transcription factors. For example, synovial cell proliferation is enhanced through the suppression of p21 and overexpression of the transcription factor c-fos [42], while mitotic activity is inhibited through wee-1 kinase [43]. This is a characteristic feature of synovial cells, representing "tumor cell-like proliferation." Indeed when $c$-fos transgenic mice are used in an arthritis model, joint destruction proceeds not as a result of lymphocyte invasion, but by synovial cell proliferation [44]. And, as reported earlier, the expression wee-1 kinase, a G2 /M cell cycle control factor, is increased in mice lacking an essential mammalian timekeeping gene $C R Y$ [45].

\section{Clock Genes and Arthritis; Cry, Per and TNF- $\alpha$ Exacerbate Inflammation}

To verify the significance of increased wee-1 expression in $C R Y$ knockout mice $\left(C R Y 1^{-/-} C R Y 2^{-/-}\right.$mice), we utilized a mouse model of arthritis [46]. First, we confirmed the influence of arthritis on clock gene expression using wild type (WT) mice that had been administered anticollagen antibody and LPS and PER2 protein levels in the synovium were monitored. PER2 is usually expressed at night, but in the arthritis model PER2 was highly expressed in the morning. In addition, the phase of PER1/2 mRNA expression in spleen lymphocytes was shifted back $\sim 6 \mathrm{hrs}$, and BMAL1 and Per1/2 mRNA expression levels were reduced. These observations indicate that the onset of arthritis indeed affects the expression of clock genes in vivo.

Next, we examined spleen-derived lymphocytes from CRY knockout mice. Peripheral T lymphocytes were found to be constitutively activated, and stimulation of splenocytes by anti-CD3/CD28 antibodies produced higher amounts of TNF- $\alpha$. In addition, wee-1 protein was overexpressed in the spleens of CRY knockout mice. Together, these results suggest that $C R Y$ knockout mice are ready or primed for arthritis onset. Accordingly, arthritis of the limbs was strongly induced by type II collagen cocktail in CRY knockout mice, and this arthritis was suppressed by anti-TNF- $\alpha$ antibodies. Finally, mutual regulation between the TNF- $\alpha$ and CRY genes was demonstrated using luciferase reporter assays.

In addition, recent studies have shown that the absence of $C R Y$ leads to constitutive activation of protein kinase A, which results in phosphorylation of p65, and thereby ultimately induces NF- $\kappa \mathrm{B}$ activation and expression of IL-6 as well as TNF- $\alpha$ [47]. Consistently, expression of CRY1 was markedly decreased by administration of melatonin, subsequently aggravated in mouse antitype II collagen antibodyinduced arthritis [48]. Further, a phase and amplitude of the clock genes were different between osteoarthritis fibroblastlike synovial cells (OA-FLS) and RA-FLS cells [49]. Thus, these results suggest that clock genes, such as CRY, seem to be deeply involved in both inflammation and arthritis.

Then, can the inflammatory pathways directly affect the expression of clock genes? Table 1 shows the effect of TNF- $\alpha$ on expression of clock genes in mouse and human cells. Using NIH3T3 mouse fibroblast cells, Cavadini et al. reported TNF$\alpha$ inhibited the expression of PER1/2/3, DBP, TEF, and HLF, slightly enhanced $C L O C K$ and $R E V-E R B \alpha$, and did not affect $B M A L 1$, by interfering with $\mathrm{E}$ box-mediated transcription of clock genes. And also IL-1 $\beta$, but not IL-6, seemed to have a biological effect as well as TNF- $\alpha$ [50]. Further, the early effect of TNF- $\alpha$ on expression of the PER1 gene is dependent on $\mathrm{p} 38$, mitogen-activated protein kinase (MAPK), and/or calcium signaling, whereas its effect on the DBP gene is independent of MAPK but dependent on calcium signaling [51]. In human monocytic THP-1 cells, TNF- $\alpha$ enhanced the expression of $B M A L 1$ and $C R Y 2$, whereas it decreased those of PER2, CRY1, and $R E V-E R B \alpha$ [52]. Unlike mouse cells, TNF- $\alpha$ did not affect or slightly enhanced the expression of PER1 in OAFLS, RA-FLS, and THP-1 cells $[49,52,53]$. However, TNF- $\alpha$ 
TABLE 1: Effect of TNF- $\alpha$ on the expression of clock genes in mouse NIH3T3, human OA-FLS, RA-FLS, and THP-1 cell line*.

\begin{tabular}{|c|c|c|c|c|}
\hline \multirow{3}{*}{ Clock gene } & Mouse & \multicolumn{3}{|c|}{ Human } \\
\hline & & Fibroblast & & Monocyte \\
\hline & NIH3T3 & OA-FLS & RA-FLS & THP-1 \\
\hline Bmall & Not affected & - & $\mathrm{Up}$ & Up \\
\hline Clock & Slightly up & - & Slightly up & Not affected \\
\hline Perl & Down (early 3 h: up) & Not affected & Slightly up & Slightly up \\
\hline Per2 & Down & - & Down & Down \\
\hline Per3 & Down & Down & - & - \\
\hline Cryl & Up & - & Up & Down \\
\hline Cry2 & Slightly down & - & Not affected & Up \\
\hline$D b p$ & Down & Down & Down & - \\
\hline Tef & Down & - & Down & - \\
\hline Hlf & Down & - & Down & - \\
\hline$E 4 B P 4$ & - & - & Up & - \\
\hline Rev-erb $\alpha$ & Slightly up & - & - & Down \\
\hline
\end{tabular}

* Based on results of the refer 49-53. - denotes no data. OA-FLS: osteoarthritis fibroblast-like synovial cells. RA-FLS: rheumatoid arthritis fibroblast-like synovial cells.

has an inhibitory effect on the expression of DBP and PER3 in OA-FLS cells [49]. And we observed, using RA-FLS cells, TNF- $\alpha$ also inhibited the expression of PER2 and slightly enhanced CLOCK as well, whereas it significantly increased the expression of BMAL1 and Cry1 [53]. Interestingly, in TNF- $\alpha$-stimulated RA-FLS cells, expression of DBP, TEF and $H L F$ was reduced while E4BP4 was increased, a positive and negative transcriptional regulator of $P E R 2$, respectively, suggesting another transcriptional regulation of PER2 by TNF$\alpha$ via $\mathrm{D}$ box, but not $\mathrm{E}$ box [53]. Thus, the proinflammatory cytokines such as TNF- $\alpha$ could affect the expression of clock genes through their inflammatory cascades. In addition, if we recall that PER2 knockout mice exhibit increased resistance to apoptosis in thymocytes [54], decreased expression of PER2 by TNF- $\alpha$ can also contribute to the resistance of synovial cells to apoptosis and may contribute to the tumorlike growth of the synovium.

Recently, series of observations has made increasingly clear the involvement of clock genes in the pathogenesis of arthritis. Kouri et al. confirmed that BMAL1 protein is markedly localized in the cytoplasm of RA synovium [49]. Interestingly, in mouse model of osteoarthritis (OA), circadian oscillations of PER2 transcription are reported to be significantly reduced in cartilage from aged mice [55], and the robust oscillations of PER2 transcription are confirmed in cartilage from juvenile mice [56]. These results suggest not only the similarity to human $\mathrm{OA}$ that affected individuals increases with age, but also the relation between circadian rhythms and physiology of cartilage formation, that is, also important for joint destruction mechanism in RA.

\section{Conclusion}

The past 20 years have seen dramatic progress in elucidating the genetic regulation of the body's circadian rhythm. An intricate system of intermolecular interaction modulates the circadian rhythms in cells to an approximate 24 -hour cycle, and this cycle is normally maintained and readjusted by both central and peripheral mechanisms. Maintenance of this cycle is important in maintaining a number of homeostatic functions in the body, and dysfunction of this body clock can promote a number of human diseases and pathologies. Since clock genes are highly conserved, it is not surprising that they are responsible for modulating a large number of biological functions. We expect that future research will help us understand not only the various functions of clock genes, but also the strategy to save the primary roles and functions of circadian clock for the human health which is disturbed by a number of factors in the modern society.

\section{Conflict of Interests}

The authors declare that there is no conflict of interests regarding the publication of this paper.

\section{References}

[1] C. A. Czeisler, J. F. Duffy, T. L. Shanahan et al., "Stability, precision, and near-24-hour period of the human circadian pacemaker," Science, vol. 284, no. 5423, pp. 2177-2181, 1999.

[2] J. F. Duffy, S. W. Cain, A.-M. Chang et al., "Sex difference in the near-24-hour intrinsic period of the human circadian timing system," Proceedings of the National Academy of Sciences of the United States of America, vol. 108, no. 3, pp. 15602-15608, 2011.

[3] S. Gaston and M. Menaker, "Pineal function: the biological clock in the sparrow?" Science, vol. 160, no. 3832, pp. 1125-1127, 1968.

[4] P. G. Sokolove, "Localization of the cockroach optic lobe circadian pacemaker with microlesions," Brain Research, vol. 87, no. 1, pp. 13-21, 1975.

[5] R. J. Lucas, G. S. Lall, A. E. Allen, and T. M. Brown, "How rod, cone, and melanopsin photoreceptors come together to enlighten the mammalian circadian clock," Progress in Brain Research, vol. 199, pp. 1-18, 2012. 
[6] S. M. Reppert and D. R. Weaver, "Coordination of circadian timing in mammals," Nature, vol. 418, no. 6901, pp. 935-941, 2002.

[7] H. Okamura, "Clock genes in cell clocks: roles, actions, and mysteries," Journal of Biological Rhythms, vol. 19, no. 5, pp. 388399, 2004.

[8] F. Guillaumond, H. Dardente, V. Giguère, and N. Cermakian, "Differential control of Bmall circadian transcription by REVERB and ROR nuclear receptors," Journal of Biological Rhythms, vol. 20, no. 5, pp. 391-403, 2005.

[9] S. Li and S. P. Hunger, "The DBP transcriptional activation domain is highly homologous to that of HLF and TEF and is not responsible for the tissue type-specific transcriptional activity of DBP," Gene, vol. 263, no. 1-2, pp. 239-245, 2001.

[10] S. Mitsui, S. Yamaguchi, T. Matsuo, Y. Ishida, and H. Okamura, "Antagonistic role of E4BP4 and PAR proteins in the circadian oscillatory mechanism," Genes and Development, vol. 15, no. 8, pp. 995-1006, 2001.

[11] H. R. Ueda, S. Hayashi, W. Chen et al., "System-level identification of transcriptional circuits underlying mammalian circadian clocks," Nature Genetics, vol. 37, no. 2, pp. 187-192, 2005.

[12] N. Koike, S. H. Yoo, H. C. Huang et al., "Transcriptional architecture and chromatin landscape of the core circadian clock in mammals," Science, vol. 338, no. 6105, pp. 349-354, 2012.

[13] D. P. Cardinali and P. Pévet, "Basic aspects of melatonin action," Sleep Medicine Reviews, vol. 2, no. 3, pp. 175-190, 1998.

[14] J. I. Chuang, S. S. Chen, and M. T. Lin, "Melatonin decreases brain serotonin release, arterial pressure and heart rate in rats," Pharmacology, vol. 47, no. 2, pp. 91-97, 1993.

[15] S. S. Gilbert, C. J. Van Den Heuvel, and D. Dawson, "Daytime melatonin and temazepam in young adult humans: equivalent effects on sleep latency and body temperatures," Journal of Physiology, vol. 514, no. 3, pp. 905-914, 1999.

[16] J. E. Gibbs and D. W. Ray, "The role of the circadian clock in rheumatoid arthritis," Arthritis Research \& Therapy, vol. 15, no. 1, article 205, 2013.

[17] M. Cutolo, G. J. M. Maestroni, K. Otsa et al., "Circadian melatonin and cortisol levels in rheumatoid arthritis patients in winter time: a north and south Europe comparison," Annals of the Rheumatic Diseases, vol. 64, no. 2, pp. 212-216, 2005.

[18] M. Afkhamizadeh, M. Sahebari, and S. R. Seyyed-Hoseini, "Morning melatonin serum values do not correlate with disease activity in rheumatoid arthritis: a cross-sectional study," Rheumatology International, 2014.

[19] M. Cutolo and G. J. M. Maestroni, "The melatonin-cytokine connection in rheumatoid arthritis," Annals of the Rheumatic Diseases, vol. 64, no. 8, pp. 1109-1111, 2005.

[20] D. S. Rudra, U. Pal, N. C. Maiti, R. J. Reiter, and S. Swarnakar, "Melatonin inhibits matrix metalloproteinase-9 activity by binding to its active site," Journal of Pineal Research, vol. 54, no. 4, pp. 398-405, 2013.

[21] L. A. Toth, "Sleep, sleep deprivation and infectious disease: studies in animals," Advances in Neuroimmunology, vol. 5, no. 1, pp. 79-92, 1995.

[22] K. L. Adams, O. Castanon-Cervantes, J. A. Evans, and A. J. Davidson, "Environmental circadian disruption elevates the IL6 response to lipopolysaccharide in blood," Journal of Biological Rhythms, vol. 28, no. 4, pp. 272-277, 2013.

[23] T. Lange, S. Dimitrov, and J. Born, "Effects of sleep and circadian rhythm on the human immune system: annals of the New
York Academy of Sciences," Annals of the New York Academy of Sciences, vol. 1193, pp. 48-59, 2010.

[24] H. Cakirbay, M. Bilici, O. Kavakçi, A. Cebi, M. Güler, and U. Tan, "Sleep quality and immune functions in rheumatoid arthritis patients with and without major depression," International Journal of Neuroscience, vol. 114, no. 2, pp. 245-256, 2004.

[25] R. M. Taylor-Gjevre, J. A. Gjevre, B. Nair, R. Skomro, and H. J. Lim, "Hypersomnolence and sleep disorders in a rheumatic disease patient population," Journal of Clinical Rheumatology, vol. 16, no. 6, pp. 255-261, 2010.

[26] C. E. Goodchild, G. J. Treharne, D. A. Booth, and S. J. Bowman, "Daytime patterning of fatigue and its associations with the previous night's discomfort and poor sleep among women with primary Sjögren's syndrome or rheumatoid arthritis," Musculoskeletal Care, vol. 8, no. 2, pp. 107-117, 2010.

[27] R. Westhovens, K. Van der Elst, A. Matthys, M. Tran, and I. Gilloteau, "Sleep problems in patients with rheumatoid arthritis," Journal of Rheumatology, vol. 41, no. 1, pp. 31-40, 2014.

[28] F. Wolfe, K. Michaud, and T. Li, "Sleep disturbance in patients with rheumatoid arthritis: evaluation by medical outcomes study and visual analog sleep scales," Journal of Rheumatology, vol. 33, no. 10, pp. 1942-1951, 2006.

[29] S. Puttonen, T. Oksanen, J. Vahtera et al., "Is shift work a risk factor for rheumatoid arthritis? The Finnish Public Sector study," Annals of the Rheumatic Diseases, vol. 69, no. 4, pp. 779780, 2010.

[30] M. Keller, J. Mazuch, U. Abraham et al., "A circadian clock in macrophages controls inflammatory immune responses," Proceedings of the National Academy of Sciences of the United States of America, vol. 106, no. 50, pp. 21407-21412, 2009.

[31] J. E. Gibbs, J. Blaikley, S. Beesley et al., “The nuclear receptor $\mathrm{REV}-\mathrm{ERB} \alpha$ mediates circadian regulation of innate immunity through selective regulation of inflammatory cytokines," Proceedings of the National Academy of Sciences of the United States of America, vol. 109, no. 2, pp. 582-587, 2012.

[32] S. Sato, T. Sakurai, J. Ogasawara et al., "A circadian clock gene, Rev-erb $\alpha$, modulates the inflammatory function of macrophages through the negative regulation of Ccl 2 expression," Journal of Immunology, vol. 192, no. 1, pp. 407-417, 2014.

[33] M. T. Lam, H. Cho, H. P. Lesch et al., "Rev-Erbs repress macrophage gene expression by inhibiting enhancer-directed transcription," Nature, vol. 498, no. 7455, pp. 511-515, 2013.

[34] K. D. Nguyen, S. J. Fentress, Y. Qiu, K. Yun, J. S. Cox, and A. Chawla, "Circadian gene Bmall regulates diurnal oscillations of Ly6C(hi) inflammatory monocytes," Science, vol. 341, no. 6153, pp. 1483-1488, 2013.

[35] S. Kirsch, S. Thijssen, S. Alarcon Salvador et al., “T-cell numbers and antigen-specific T-cell function follow different circadian rhythms," Journal of Clinical Immunology, vol. 32, no. 6, pp. 1381-1389, 2012.

[36] M. Cutolo and A. T. Masi, "Circadian rhythms and arthritis," Rheumatic Disease Clinics of North America, vol. 31, no. 1, pp. 115-129, 2005.

[37] R. Alten, "Chronotherapy with modified-release prednisone in patients with rheumatoid arthritis," Expert Review of Clinical Immunology, vol. 8, no. 2, pp. 123-133, 2012.

[38] M. Cutolo, "Chronobiology and the treatment of rheumatoid arthritis," Current Opinion in Rheumatology, vol. 24, no. 3, pp. 312-318, 2012.

[39] M. C. Boissier, L. Semerano, S. Challal, N. Saidenberg-Kermanac'h, and G. Falgarone, "Rheumatoid arthritis: from 
autoimmunity to synovitis and joint destruction," Journal of Autoimmunity, vol. 39, no. 3, pp. 222-228, 2012.

[40] A. Hashiramoto, C. Sakai, K. Yoshida et al., "Angiopoietin 1 directly induces destruction of the rheumatoid joint by cooperative, but independent, signaling via ERK/MAPK and phosphatidylinositol 3-kinase/Akt," Arthritis and Rheumatism, vol. 56, no. 7, pp. 2170-2179, 2007.

[41] N. Komatsu and H. Takayanagi, "Autoimmune arthritis: the interface between the immune system and joints," Advances in Immunology, vol. 115, pp. 45-71, 2012.

[42] M. Hikasa, E. Yamamoto, H. Kawasaki et al., "p21waf1/cip1 is down-regulated in conjunction with up-regulation of c-Fos in the lymphocytes of rheumatoid arthritis patients," Biochemical and Biophysical Research Communications, vol. 304, no. 1, pp. 143-147, 2003.

[43] H. Kawasaki, K. Komai, M. Nakamura et al., "Human weel kinase is directly transactivated by and increased in association with c-Fos/AP-1: rheumatoid synovial cells overexpressing these genes go into aberrant mitosis," Oncogene, vol. 22, no. 44, pp. 6839-6844, 2003.

[44] S. Shiozawa, Y. Tanaka, T. Fujita, and T. Tokuhisa, "Destructive arthritis without lymphocyte infiltration in $\mathrm{H} 2$-c-fos transgenic mice," Journal of Immunology, vol. 148, no. 10, pp. 3100-3104, 1992.

[45] T. Matsuo, S. Yamaguchi, S. Mitsui, A. Emi, F. Shimoda, and H. Okamura, "Control mechanism of the circadian clock for timing of cell division in vivo," Science, vol. 302, no. 5643, pp. 255-259, 2003.

[46] A. Hashiramoto, T. Yamane, K. Tsumiyama et al., "Mammalian clock gene Cryptochrome regulates arthritis via proinflammatory cytokine TNF- $\alpha$," Journal of Immunology, vol. 184, no. 3, pp. 1560-1565, 2010.

[47] R. Narasimamurthy, M. Hatori, S. K. Nayak, F. Liu, S. Panda, and I. M. Verma, "Circadian clock protein cryptochrome regulates the expression of proinflammatory cytokines," Proceedings of the National Academy of Sciences of the United States of America, vol. 109, no. 31, pp. 12662-12667, 2012.

[48] J. Bang, H. W. Chang, H.-R. Jung et al., "Melatonin attenuates clock gene cryptochromel, which may aggravates mouse antitype II collagen antibody-induced arthritis," Rheumatology International, vol. 32, no. 2, pp. 379-385, 2012.

[49] V. P. Kouri, J. Olkkonen, E. Kaivosoja et al., "Circadian timekeeping is disturbed in rheumatoid arthritis at molecular level," PLoS ONE, vol. 8, no. 1, Article ID e54049, 2013.

[50] G. Cavadini, S. Petrzilka, P. Kohler et al., "TNF- $\alpha$ suppresses the expression of clock genes by interfering with E-box-mediated transcription," Proceedings of the National Academy of Sciences of the United States of America, vol. 104, no. 31, pp. 12843-12848, 2007.

[51] S. Petrzilka, C. Taraborrelli, G. Cavadini, A. Fontana, and T. Birchler, "Clock gene modulation by TNF- $\alpha$ depends on calcium and p38 MAP kinase signaling," Journal of Biological Rhythms, vol. 24, no. 4, pp. 283-294, 2009.

[52] M. Perez-Aso, J. L. Feig, A. Mediero, and B. N. Cronstein, "Adenosine A2A receptor and TNF- $\alpha$ regulate the circadian machinery of the human monocytic THP-1 cells," Inflammation, vol. 36, no. 1, pp. 152-162, 2013.

[53] K. Yoshida, A. Hashiramoto, T. Okano, T. Yamane, N. Shibanuma, and S. Shiozawa, "TNF- $\alpha$ modulates expression of the circadian clock gene Per2 in rheumatoid synovial cells," Scandinavian Journal of Rheumatology, vol. 42, no. 4, pp. 276280, 2013.
[54] L. Fu, H. Pelicano, J. Liu, P. Huang, and C. C. Lee, “The circadian gene Period2 plays an important role in tumor suppression and DNA damage response in vivo," Cell, vol. 111, no. 1, pp. 41-50, 2002.

[55] N. Gossan, L. Zeef, J. Hensman et al., "The circadian clock in murine chondrocytes regulates genes controlling key aspects of cartilage homeostasis," Arthritis \& Rheumatology, vol. 65, no. 9, pp. 2334-2345, 2013.

[56] N. Okubo, Y. Minami, H. Fujiwara et al., "Prolonged bioluminescence monitoring in mouse ex vivo bone culture revealed persistent circadian rhythms in articular cartilages and growth plates," PLoS ONE, vol. 8, no. 11, Article ID e78306, 2013. 


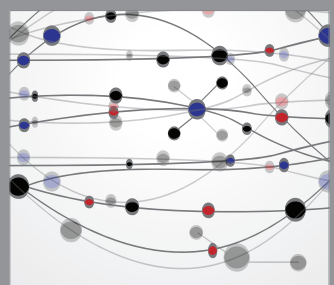

The Scientific World Journal
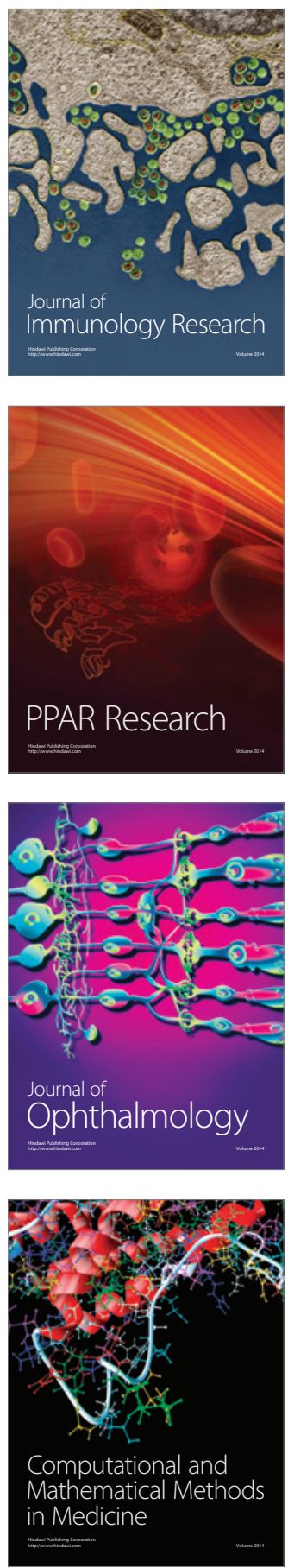

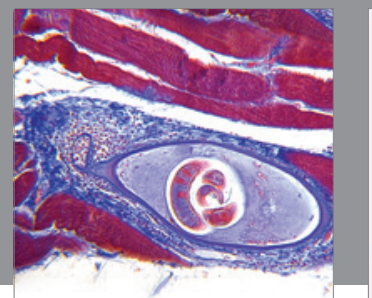

Gastroenterology

Research and Practice
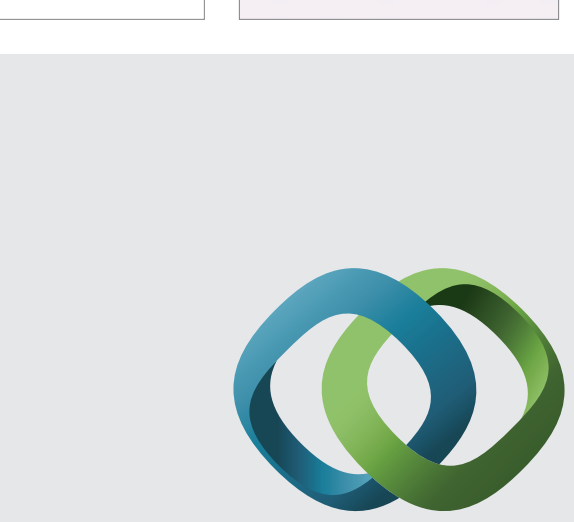

\section{Hindawi}

Submit your manuscripts at

http://www.hindawi.com
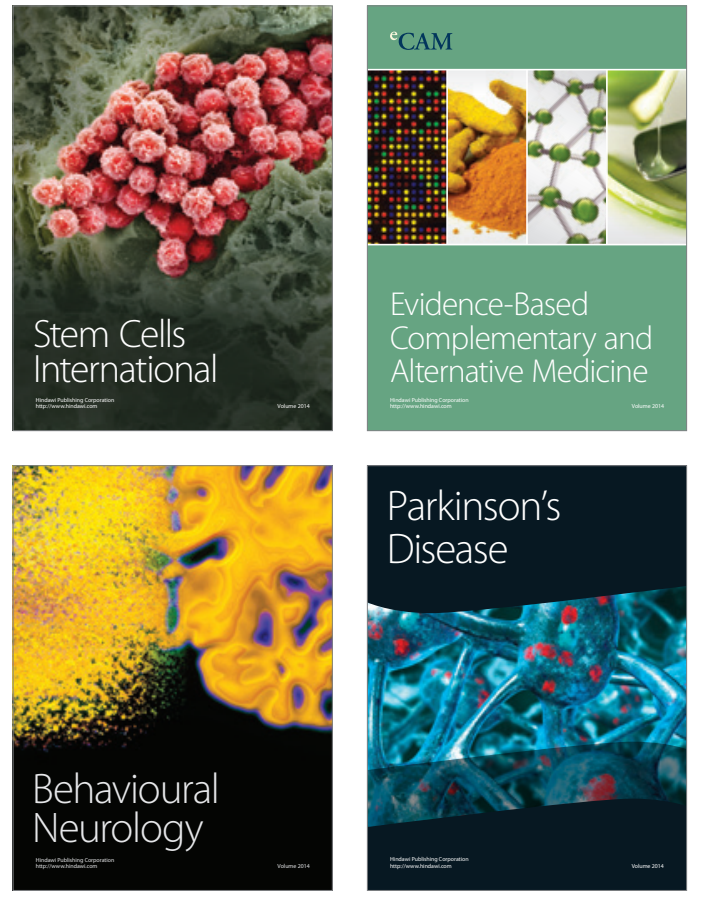
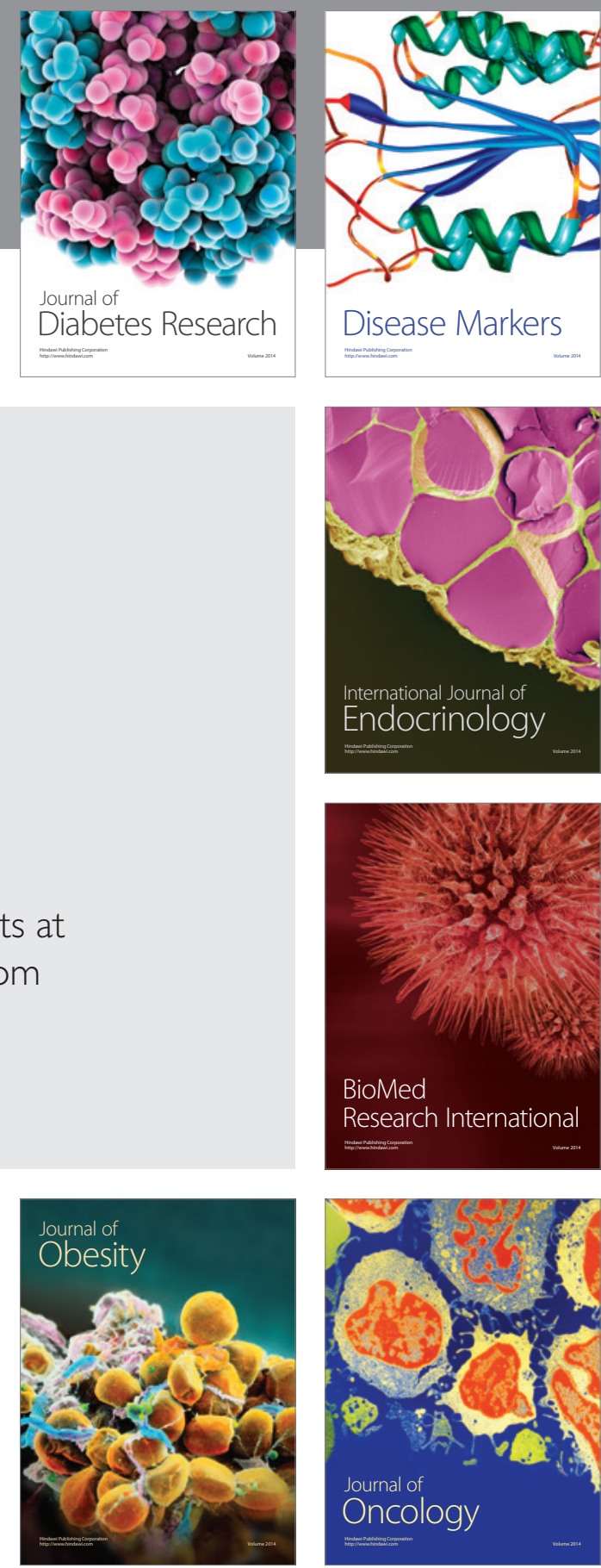

Disease Markers
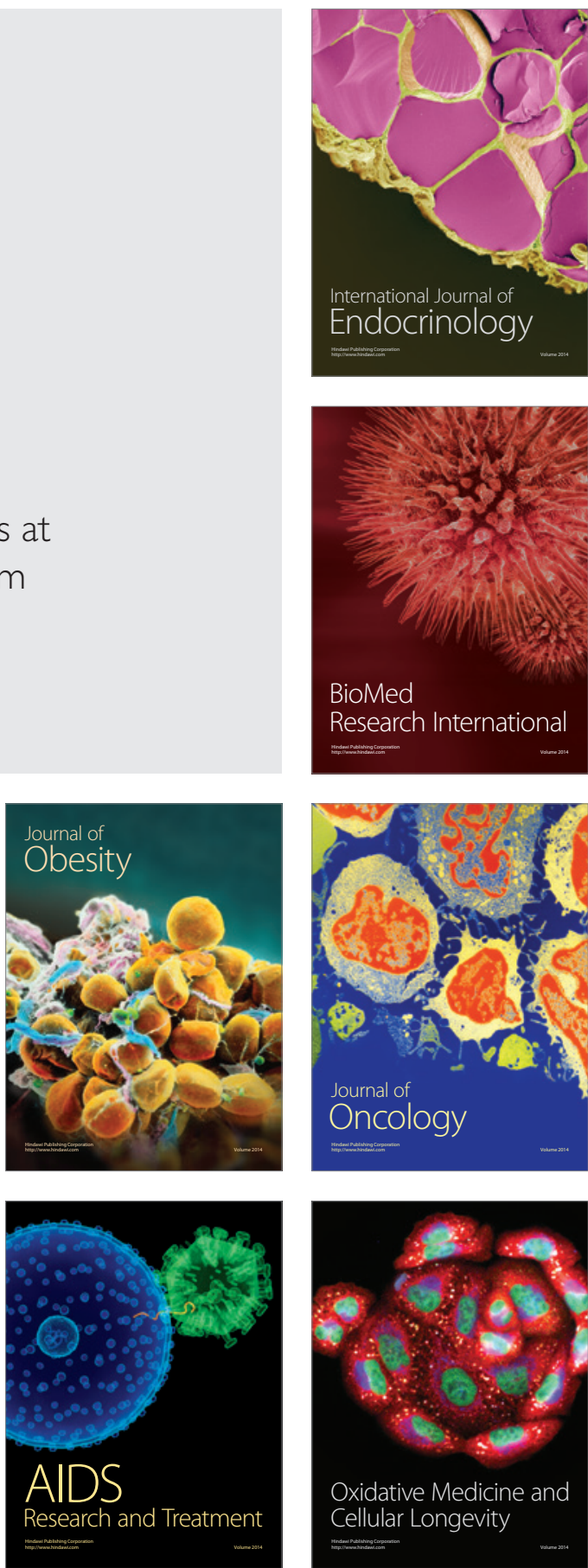